INPLASY

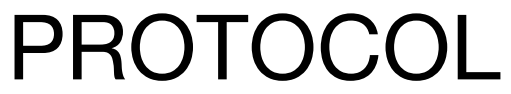

To cite: Huang et al.

Psychological effects of traditional Chinese mind-body exercises for low back pain: a protocol for meta-analysis. Inplasy protocol 202130075. doi:

10.37766/inplasy2021.3.0075

Received: 21 March 2021

Published: 21 March 2021

Corresponding author:

Peiguo Zhang

drzhang0318@163.com

Author Affiliation:

ZiBo central hospital

Support: Lianyungang Hospital of TCM.

Review Stage at time of this submission: The review has not yet started.

Conflicts of interest: None declared.

\section{Psychological effects of traditional Chinese mind-body exercises for low back pain: a protocol for meta- analysis}

\author{
Huang, Q1; Du, J2; Zhang, X³.
}

Review question / Objective: What are psychological effects of traditional Chinese mind-body exercises for low back pain? P: patients with low back pain. I: traditional Chinese mindbody exercises. C: it will be compared with any other. $O$ : psychological effects, such as anxiety, depression, etc.

Condition being studied: Several studies reported that traditional Chinese mind-body exercises showed beneficial effects on improving anxiety and depression of patients with low back pain in recent years. However, the effects of traditional Chinese mind-body exercises on improving psychological disorders of patients with low back pain remain controversial. Most previous reviews only focused on the effects of traditional Chinese mind-body exercises for low back pain on pain and dysfunction. Therefore, the present systematic review and meta-analysis will be conducted to evaluate the evidence on psychological effects of traditional Chinese mind-body exercises for low back pain.

INPLASY registration number: This protocol was registered with the International Platform of Registered Systematic Review and Meta-Analysis Protocols (INPLASY) on 21 March 2021 and was last updated on 21 March 2021 (registration number INPLASY202130075).

\section{INTRODUCTION}

Review question / Objective: What are psychological effects of traditional Chinese mind-body exercises for low back pain? P: patients with low back pain. I: traditional Chinese mind-body exercises. C: it will be compared with any other. O: psychological effects, such as anxiety, depression, etc.

Condition being studied: Several studies reported that traditional Chinese mindbody exercises showed beneficial effects on improving anxiety and depression of 
patients with low back pain in recent years. However, the effects of traditional Chinese mind-body exercises on improving psychological disorders of patients with low back pain remain controversial. Most previous reviews only focused on the effects of traditional Chinese mind-body exercises for low back pain on pain and dysfunction. Therefore, the present systematic review and meta-analysis will be conducted to evaluate the evidence on psychological effects of traditional Chinese mind-body exercises for low back pain.

\section{METHODS}

Search strategy: The electronic databases (PubMed, Embase, MEDLINE, Cochrane Central Register of Controlled Trials, Web of Science, China Knowledge Resource Integrated Database, and Wanfang Data) will be searched. The search will include all articles from their inception to February 2021. The search terms will be used, as follows: (1) "low back pain" or "lumbago" or "Iumbar disc herniation" or "lumbar sprain" or "backache"; (2) "Tai Chi" or "Tai Chi Chuan" or "Qigong" or "Baduanjin" or "Wuqinxi" or "Yijinjing" and (3) "psychological effects", or "anxiety", or "depression".

Participant or population: Studies will be eligible if they include participants with a diagnosis of low back pain. There were no limitations on age, gender, or nationality of patients with low back pain.

Intervention: Traditional Chinese mindbody exercises include Tai Chi, Qigong, Baduanjin, Wuqinxi, and Yijinjing.

Comparator: The control interventions include medicine, observation, manual therapy, acupuncture, traction, education, and any treatments without traditional Chinese mind-body exercises. The study assessing the effects of traditional Chinese mind-body exercises plus an intervention compared with the same intervention (such as Tai Chi plus traction versus traction) will also be included.
Study designs to be included: Only randomized control trials will be included.

Eligibility criteria: The randomized control trials assessed the effects of traditional Chinese mind-body exercises for low back pain on psychological effects such as anxiety, depression, etc.

Information sources: The electronic databases (PubMed, Embase, MEDLINE, Cochrane Central Register of Controlled Trials, Web of Science, China Knowledge Resource Integrated Database, and Wanfang Data) will be searched. The search will include all articles from their inception to February 2021.

Main outcome(s): The main outcomes will include psychological well-being such as depression, anxiety, etc.

Additional outcome(s): Additional outcomes include pain, disability, and quality of life.

Quality assessment / Risk of bias analysis: The quality assessment of the included studies will be independently conducted by two reviewers using the Physiotherapy Evidence Database (PEDro) scale. Quality of evidence will be assessed using the Grades of Recommendation, Assessment, Development and Evaluation.

Strategy of data synthesis: The metaanalysis will be conducted using Review Manager Version $\mathbf{5 . 3}$ software. For continuous data, the change between baseline and the end of interventions will be used in the meta-analysis. The mean difference (MD) and $95 \%$ confidence intervals (Cl) will be calculated. In the case of different outcome measure scales, the standardized mean difference (SMD) and $95 \% \mathrm{Cl}$ will be calculated. For the expected heterogeneity, the continuous data will be pooled using a more conservative randomeffects model. According to the recommendations of the Cochrane handbook for systematic reviews of interventions, the heterogeneity will be assessed using 12 statistic. It will be considered to be statistically significant 
when $P<0.10$. An Egger's test will be performed to examine publication bias.

Subgroup analysis: The subgroup analysis will be conducted based on different traditional Chinese mind-body exercises.

Sensitivity analysis: Sensibility analysis will be performed by evaluating the changes of pooled effect size after each study was eliminated one by one.

Country(ies) involved: China.

Keywords: Mind-body exercises, low back pain, psychological effects.

Contributions of each author:

Author 1 - Peiguo Zhang.

Author 2 - Juan Du.

Author 3 - Qian Huang. 